\title{
Psoriasiform drug eruption provoked by oriental herbal decoction 'Hyeonggaeyeongyotang'
}

\section{Osung Kwon, Hyun Chung, Joonsoo Park}

\author{
Department of Dermatology, School of Medicine, Catholic University of Daegu, Namgu, Daegu, South Korea
}

Corresponding author: Joonsoo Park, M.D., Ph.D., E-mail: ashkwon@naver.com

Sir,

Specialization of treatment in traditional Korean medicine has increased the number of patients seeking traditional medicine, as well as in the frequency of related adverse events. There are currently several medical issues in Korea, including rational issues, due to the use of traditional medicine without the approval of the Korean Food Drug Administration. Herein, we present a case of psoriasiform drug eruption that developed after ingesting a medicinal decoction prepared at a clinic without precise standards for ingredients, method of use, or dosage.

A 5-year-old female patient presented with papules on both knees that accompanied with pruritus, and had insidiously spread for one month. Numerous skin-colored hyperkeratotic slightly annular-shaped papules were elucidated on both knees (Fig. 1). Some of the papules were observed to form clusters. Koebner phenomenon and Auspitz's sign was not present. Two months in prior, the patient visited an oriental clinic to treat rhinitis and was prescribed to take hyeonggaeyeongyotang, an herbal decoction, every day for one month. The patient was generally in good health with no specific family history of skin diseases. Laboratory tests were nonspecific except for elevated liver enzyme. Skin biopsy was performed on the left knee and revealed parakeratosis ith variably elongated epidermis and superficial perivascular infiltration of lymphocytes (Fig. 2a). Higher magnification revealed few eosinophils and flattened granular layer (Fig. 2b). The patient was diagnosed with psoriasiform drug eruption, and was instructed to halt previous decoction. After one week, the lesions began to improve and significantly recovery was noticed after three weeks (Fig. 3).

Psoriasiform drug eruption is a general term for diseases that occur after medication and presents clinical and histological traits similar to psoriasis. Diagnosis is based on histology and a causal relation of medication use is crucial. The disease occurs after medication and improves after stopping medication. The distribution of lesions and scaling are known to be milder than psoriasis. Koebner phenomenon and Auspitz's sign are known to be less common in psoriasiform drug eruption [1].

The relationship between drug and cutaneous eruption was assessed accordingly to the criteria set by and the present case scored a total of 6 points, indicative

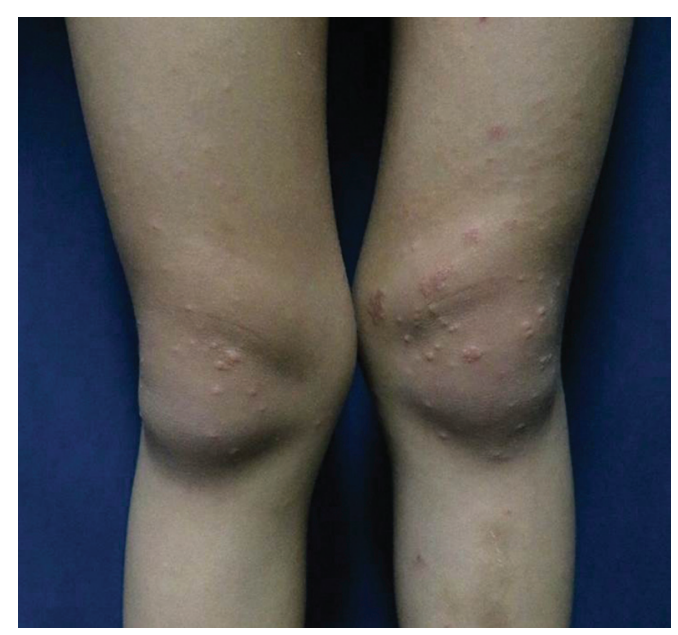

Figure 1: Clinical image on initial visit displaying numerous skin colored hyperkeratotic papules Three months after discontinuing of the drug shows improved skin lesion.

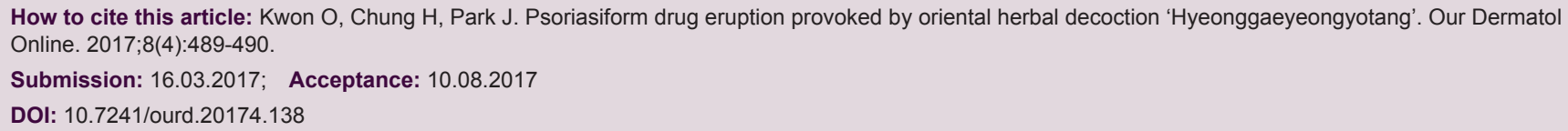




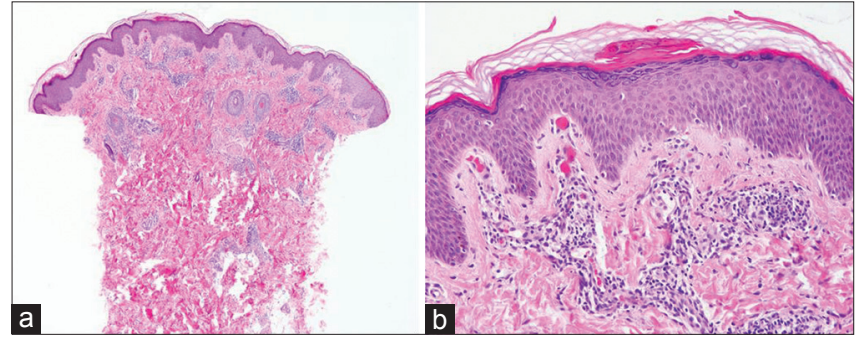

Figure 2: a) Histology revealed irregular acanthosis, focal parakeratosis with variably elongated epidermis and superficial perivascular infiltration of lymphocytes (H\&E x20). b) Higher magnification revealed extravasation of clumped erythrocytes with few eosinophils (H\&E x200).

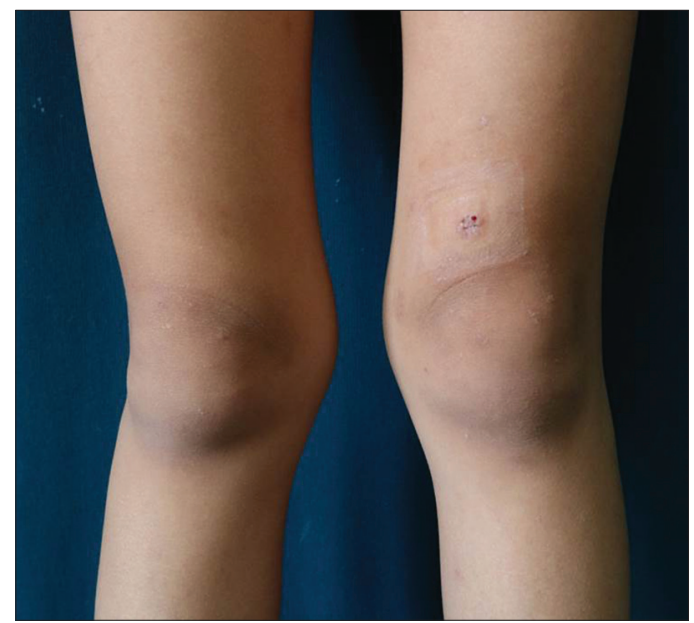

Figure 3: Three months after discontinuing of the drug shows improved skin lesion with noticeably regressed grouped skin colored papules leaving subtle hyperpigmentation.

of a high correlation [2]. The rapid response upon discontinuing the drug along with no other causative factors further supplements a high association between the herbal medication and cutaneous eruption $[1,2]$.

Copious number of adverse effects is being reported after administration of traditional Korean medicine. Kim et al reported that among these event, skin rash was the second most common side effect followed by gastrointestinal symptoms from herbal medications [3]. The patient in our case was prescribed with Hangyeyeongyotang and the ingredients in the prescription contained herbs such as hyeonggae (Schizonepeta tenuifolia), yeongyo (Forsythia viridissima), jisil (Poncirus trifoliata), gilgyeong (Platycodon grandiflorum), gamcho (Glycyrrhiza glabra), danggwi (Angelica gigas), jagyak (Paeonia lactiflora), and cheongung (Cnidium officiale). Combining research of these individual ingredients report hepatotoxicity and non-specific skin eruption which was seen in our patient, further suggests likelihood of psoriasiform drug eruption $[4,5]$.

No single case reporting an association between herbal medicine and psoriasiform drug eruption has been reported. Lack of accurate research due to the current system, wherein herbal medicines are prescribed without criteria for ingredients, method of use, dosing may hinder further elaboration. This case underlines the importance of drug ingredient analysis to avoid recurrence and difficulties in treatment and finding the cause of the eruption.

\section{REFERENCES}

1. Rambhia KD, Gulati AS, Pande S. Psoriasis versus psoriasiform drug eruption. Indian J Drugs Dermatol. 2016;2:48-9.

2. Dika E, Varotti C, Bardazzi F, Maibach HI. Drug-induced psoriasis: an evidence-based overview and the introduction of psoriatic drug eruption probability score. Cutan Ocul Toxicol. 2006;25:1-11.

3. Kim M, Han C. Analysis of Herbal-drug-associated Adverse Drug Reactions Using Data from Spontaneous Reporting System in Electronic Medical Reords. J Korean Med. 2015;36:45-60.

4. Kim DM, Han HK, Cho SY. Retrospective Observation of Liver Function Parameters for 101 Patients Using Herbal Drugs for one month. J Korean Oriental Med. 2010;31:149-57.

5. Parker S, Zhang CS, Yu JJ. Oral Chinese herbal medicine versus placebo for psoriasis vulgaris: A systemic review. J Dermatolog Treat. 2016;1:1-11.

Copyright by Osung Kwon, et al. This is an open-access article distributed under the terms of the Creative Commons Attribution License, which permits unrestricted use, distribution, and reproduction in any medium, provided the original author and source are credited.

Source of Support: Nil, Conflict of Interest: None declared. 